\title{
Fractionated photothermal antitumor therapy with multidye nanoparticles
}

\author{
This article was published in the following Dove Press journal: \\ International Journal of Nanomedicine \\ 19 January 2012 \\ Number of times this article has been viewed
}

\author{
Luke G Gutwein' \\ Amit K Singh ${ }^{2}$ \\ Megan A Hahn ${ }^{2}$ \\ Michael C Rule ${ }^{3}$ \\ Jacquelyn A Knapik ${ }^{4}$ \\ Brij M Moudgil ${ }^{2}$ \\ Scott C Brown ${ }^{2}$ \\ Stephen R Grobmyer \\ 'Division of Surgical Oncology, \\ Department of Surgery, College \\ of Medicine, ${ }^{2}$ Particle Engineering \\ Research Center, ${ }^{3} \mathrm{Cell}$ and Tissue \\ Analysis Core, McKnight Brain \\ Institute, ${ }^{4}$ Department of Pathology, \\ University of Florida, Gainesville, \\ FL, USA
}

Correspondence: Stephen R Grobmyer Department of Surgery, University of Florida, I600 SW Archer Road, Room 6164, PO Box 100109 ,

Gainesville, FL 32610-0109, USA

Tel +l 3522650169

Fax +I 3522651060

Email stephen.grobmyer@surgery.ufl.edu
Purpose: Photothermal therapy is an emerging cancer treatment paradigm which involves highly localized heating and killing of tumor cells, due to the presence of nanomaterials that can strongly absorb near-infrared (NIR) light. In addition to having deep penetration depths in tissue, NIR light is innocuous to normal cells. Little is known currently about the fate of nanomaterials post photothermal ablation and the implications thereof. The purpose of this investigation was to define the intratumoral fate of nanoparticles (NPs) after photothermal therapy in vivo and characterize the use of novel multidye theranostic NPs (MDT-NPs) for fractionated photothermal antitumor therapy.

Methods: The photothermal and fluorescent properties of MDT-NPs were first characterized. To investigate the fate of nanomaterials following photothermal ablation in vivo, novel MDTNPs and a murine mammary tumor model were used. Intratumoral injection of MDT-NPs and real-time fluorescence imaging before and after fractionated photothermal therapy was performed to study the intratumoral fate of MDT-NPs. Gross tumor and histological changes were made comparing MDT-NP treated and control tumor-bearing mice.

Results: The dual dye-loaded mesoporous NPs (ie, MDT-NPs; circa $100 \mathrm{~nm}$ ) retained both their NIR absorbing and NIR fluorescent capabilities after photoactivation. In vivo MDT-NPs remained localized in the intratumoral position after photothermal ablation. With fractionated photothermal therapy, there was significant treatment effect observed macroscopically $(P=0.026)$ in experimental tumor-bearing mice compared to control treated tumor-bearing mice.

Conclusion: Fractionated photothermal therapy for cancer represents a new therapeutic paradigm enabled by the application of novel functional nanomaterials. MDT-NPs may advance clinical treatment of cancer by enabling fractionated real-time image guided photothermal therapy.

Keywords: fluorescence, mesoporous silica, biodistribution, photothermal ablation, live animal imaging, near-infrared nanoparticle, breast cancer

\section{Introduction}

Photothermal ablation is an exciting noninvasive cancer paradigm, ${ }^{1-5}$ which is just beginning to enter the clinical arena for human cancer therapy. ${ }^{6}$ Numerous nanomaterials including nanoshells, ${ }^{1,2}$ polyhydroxy fullerenes, ${ }^{5}$ gold speckled silica nanoparticles (NPs),${ }^{7}$ gold NPs,${ }^{8}$ gold nanorods,${ }^{3}$ nanotubes,${ }^{9,10}$ nanocages, ${ }^{11}$ and quantum dots ${ }^{12}$ have been investigated as anticancer photothermal agents. Numerous studies have demonstrated the efficacy of these nanomaterials for tumor ablation in vitro. ${ }^{2,7,8,13}$ Several studies have focused on the effect of photothermal therapy using these nanomaterials using in vivo models. ${ }^{1,10,13}$ These in vivo studies have demonstrated that a single photothermal treatment results in partial but incomplete tumor destruction based on long 
term follow-up of tumor growth. ${ }^{10,13}$ Approaches and strategies to improve the efficacy of the photothermal antitumor strategy are needed.

Little is known about the biodistribution of photothermally active NPs following photothermal tumor ablation. Understanding the intratumoral fate of nanomaterials after photothermal therapy is essential for furthering the clinical application of nanoparticle-mediated photothermal therapy. To investigate this issue in vivo requires the development and application of nanomaterials that are not only robust photothermal reagents, but also stable bioimaging contrast agents. Magnetic resonance imaging, ${ }^{1}$ optical coherence tomography, ${ }^{13}$ and photoacoustic imaging ${ }^{14}$ have been used to guide photothermal treatments in vivo. Near-infrared (NIR) fluorescence imaging has not been utilized commonly to track photothermal nanomaterials in vivo due to difficulties in combining plasmonic materials with a fluorescent component into single, biocompatible nanoconstructs. ${ }^{15}$

In this report, novel multidye theranostic NPs (MDT-NPs) were employed to address the fate of nanomaterials following photothermal ablation. These recently developed NPs are biocompatible, have strong and stable NIR fluorescence, and robust NIR photothermal properties. ${ }^{16}$ The unique multifunctionality and stability of these novel nanomaterials favor their application to answer these questions regarding the fate of nanomaterials of photothermal therapies. In this report, the ability of MDT-NPs to mediate enhanced photothermal tumor destruction when utilized for multiple fractionated ablations is demonstrated. The findings suggest fractionated photothermal therapy as a new paradigm for noninvasive image-guided cancer therapy.

\section{Materials and methods}

NIR fluorescent (NIRF) mesoporous silica NPs ${ }^{16}$ were synthesized by incorporating a modified, silane-conjugated heptamethine cyanine dye (IR780; Sigma-Aldrich Corporation, St Louis, MO) during the synthesis of mesoporous silica NPs, as described elsewhere. ${ }^{16}$ Briefly, $0.5 \mathrm{~g}$ of cetyltrimethylammonium bromide $\left(\mathrm{C}_{16} \mathrm{TAB}\right.$; Sigma-Aldrich) and $1.75 \mathrm{~mL}$ of $2 \mathrm{M}$ sodium hydroxide (Fisher Scientific Co, Pittsburgh, PA) were added to $240 \mathrm{~mL}$ of Nanopure water (Fisher Scientific) and heated to $80^{\circ} \mathrm{C}$. Once thermal equilibrium was reached, $2.5 \mathrm{~mL}$ of tetraethyl orthosilicate (Fisher Scientific) was added dropwise to this solution. After 2-3 minutes, the transparent solution turned milky due to the nucleation of silica NPs. At this time, $800 \mu \mathrm{L}$ of silane conjugated dye was added. The particle suspension was allowed to stir for 3 hours at constant temperature of $80^{\circ} \mathrm{C}$.
For the removal of surfactant, particles were dispersed in $3 \mathrm{wt} \%$ methanolic solution of calcium chloride (Fisher Scientific) and the suspension was refluxed for 4 hours at $60^{\circ} \mathrm{C}$. For $1 \mathrm{~g}$ particles, $100 \mathrm{~mL}$ of calcium chloride-methanol solution was used. These particles were centrifuged $(7000 \times \mathrm{g}$, 20 min; Beckman Model J2-21, APE-Bridgepath Scientific, Frederick, MD) and resuspended in the calcium chloridemethanol solution with the aid of sonication (Crest Tru-Sweep Model 575D; Crest Ultrasonics, Trenton, NJ). The refluxing/ centrifugation/resuspension steps were repeated three times in order to completely remove the surfactant from the pores. Finally, these particles were suspended in Nanopure water by centrifugation and resuspension using sonication. To confirm the complete removal of surfactant, the NPs were incubated with cells and a lactate dehydrogenase assay (Roche Diagnostics, Indianapolis, IN) was performed; the assay demonstrated no deleterious effects on cells.

For the synthesis of MDT-NPs, NIRF mesoporous particles were dispersed in chloroform (Fisher Scientific) to encapsulate the photothermal dye inside the pores. In a typical synthesis, NIRF mesoporous silica NPs (10 mg) were dispersed in $8 \mathrm{~mL}$ chloroform. Then, $2 \mathrm{mg}$ of silicon 2,3-naphthalocyanine dihydroxide (Sigma-Aldrich) in chloroform (concentration $1 \mathrm{mg} / \mathrm{mL}$ ) was added to the nanoparticle dispersion. The dispersion was stirred overnight to allow for the maximum loading of the dyes into the NP pores. Particles were washed by centrifugation and were redispersed in water for further experiments. Measuring the absorbance of the supernatant allowed an estimate of the amount of dye incorporated inside the pores of the NPs (11.1\%). Nanoparticles were characterized for absorbance, emission, and heating using techniques detailed elsewhere. ${ }^{16}$

The 4T1 murine mammary tumor cell line (Tufts University, Medford, MA) was orthotopically implanted $\left(1 \times 10^{6}\right.$ cells) into female 6-week-old BALB/c mice (Charles River Laboratories, Wilmington, MA). On postimplantation day eight, 27 mice were equally and randomly distributed to nine groups (three mice per group): MDT-NP/no ablation, MDT-NP/one ablation, MDT-NP/two ablations, MDT-NP/ three ablations, MDT-NP/four ablations, control/one ablation, control/two ablations, control/three ablations, control/four ablations. Only one ablation was performed per day per mouse. At the start of the experiment, mice were initially imaged via fluorescence (710 nm excitation/820 nm emission) with the IVIS $^{\circledR}$ Spectrum (Caliper Life Sciences, Hopkinton, MA). For in vivo imaging experiments, $820 \mathrm{~nm}$ wavelength was chosen as it provides the greatest signal to noise ratio.

This was followed by administration of MDT-NPs via intratumoral injection $(15 \mathrm{mg} / \mathrm{mL}, 20 \mu \mathrm{L})$ or $0.9 \%$ sterile 
saline solution ( $20 \mu \mathrm{L}$; Fisher Scientific) for control mice. Fluorescence imaging was obtained on all mice after intratumoral injection. Mice then underwent photothermal ablation (continuous wave laser, $785 \mathrm{~nm}, 625 \mathrm{~mW} / \mathrm{cm}^{2}, 5$ minute duration, $1.5 \mathrm{~cm}$ source-tumor distance). Fluorescence imaging was also obtained after each photothermal ablation.

For imaging and ablation experiments, mice were anesthetized using 2\%-3\% isoflurane (Baxter Healthcare Corporation, Deerfield, IL). On day nine, whole animal digital photographs (D90; Nikon, Melville, NY) were obtained before mice were euthanized. Severity of treatment effect was based on gross tumor inspection (experiment day nine) and scored in a blinded fashion using a scale of zero (no treatment) to four (maximal treatment effect). After euthanization, tumors were harvested and in situ organ fluorescence images were obtained. Tumors were preserved in $10 \%$ neutral buffered formalin (Fisher Scientific) for histological analysis. Histological tumor analysis was performed in a blinded fashion by a board certified pathologist (JAK). Region of interest quantification of fluorescent signal was performed at all imaging points with Living Image ${ }^{\circledR} 3.2$ or 4.0 software (Caliper Life Sciences).

Statistical analysis was performed on the severity of tumor necrosis using the Kruskal-Wallis test (SAS Institute, Inc, Cary, NC). $P<0.05$ was considered statistically significant. All experiments were performed under protocols approved by the University of Florida Institutional Animal Care and Use Committee.

\section{Results}

MDT-NPs were synthesized using a two-step approach. ${ }^{16}$ First, NIRF mesoporous silica NPs were synthesized by incorporating a modified, silane-conjugated heptamethine cyanine dye (IR780) during the surfactant-templated synthesis of mesoporous silica NPs. Second, for the synthesis of MDT-NPs, these NIRF mesoporous NPs were dispersed in chloroform to encapsulate the NIR photothermal silicon 2,3-naphthalocyanine dihydroxide dye inside the pores of the silica matrix. The final nanoconstructs were washed and dispersed in water for the subsequent sequential photothermal ablation experiments and visualization.

The photophysical properties (absorbance and fluorescence) of NIRF NPs are presented in Figure 1A. The broad excitation spectra and large Stoke's shift of the particles enabled use of a broad range of excitation wavelengths and decreased the extent of self-quenching, respectively. Having a porous interior structure, these NPs were circa $100 \mathrm{~nm}$, as measured by transmission electron microscope (JEOL 2010F, Tokyo, Japan) (Figure 1D). Upon loading of a silicon 2,3-naphthalocyanine dihydroxide
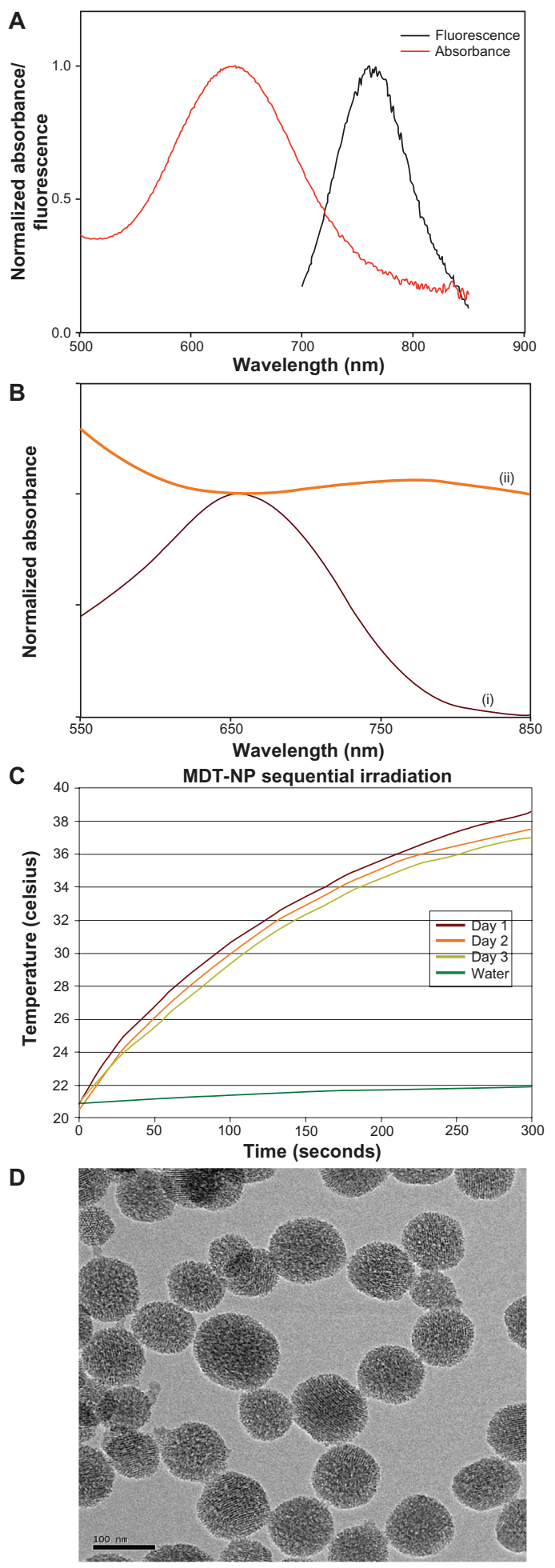

Figure I (A) Normalized absorbance and fluorescence of multidye theranostic nanoparticles. (B) Normalized ultraviolet-visible absorbance spectra of nanoparticles before (i) and after (ii) loading of silicon 2,3-naphthalocyanine dihydroxide dye. (C) Thermal properties of multidye theranostic nanoparticles. The 785-nm laser irradiated the same aqueous dispersion of multidye theranostic nanoparticles $(\mathrm{l} \mathrm{mg} / \mathrm{mL})$ on three separate days demonstrating that the multidye theranostic nanoparticles retain their photothermal properties after activation with near-infrared light. (D) Transmission electron micrograph of multidye theranostic nanoparticles. The porous structure of particles is evident in the image.

Note: Scale bar represents $100 \mathrm{~nm}$.

Abbreviation: MDT-NP, multidye theranostic nanoparticle. 
dye into the pores of NIRF NPs, their absorption cross-section in the NIR region increased (Figure 1B); the broad extinction spectra of these MDT-NPs displayed their ability to absorb light over the entire NIR region. The ability of MDT-NPs to absorb NIR light and generate heat was tested by measuring the temperature increase of an aqueous dispersion of MDT-NPs upon illumination with a low power laser source $(785 \mathrm{~nm}, 625 \mathrm{~mW} /$ $\left.\mathrm{cm}^{2}\right)$. The temperature of a solution of MDT-NPs $(1 \mathrm{mg} / \mathrm{mL})$ increased by approximately $15^{\circ} \mathrm{C}$ after 5 minutes of continuous irradiation. The repeated heating over a period of 3 days resulted in only partial loss $(\sim 4 \%$ decrease in temperature on day three relative to day one) of their heat generating capacity (Figure 1C).

Next, a murine orthotopic model of breast cancer was used to study the fate of MDT-NPs following photothermal ablation in vivo. After intratumoral injection of MDT-NPs, mammary tumors in mice were exposed to NIR laser light once daily for 1, 2, 3, or 4 days. After each NIR irradiation event, the fluorescence signal was plotted by normalizing each signal to the intensity of the original injected MDT-NPs (day one before ablation, Figure 2). Following a single injection of MDT-NPs, all experimental groups (both with and without laser irradiation) showed a consistent intratumoral fluorescence pattern for 9 days. A decrease in fluorescence signal occurred over time for each experimental group. Importantly, MDT-NPs could easily been seen in the intratumoral position for the entire 9-day observation period in all groups.

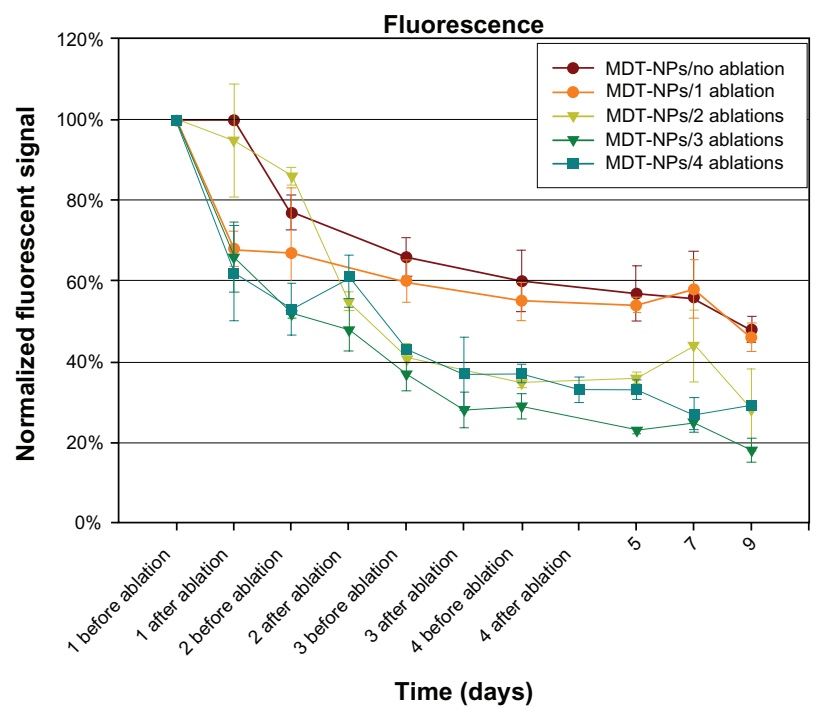

Figure 2 Nine-day time course of normalized fluorescent signal before and after photothermal ablation of control (no ablation) and sequentially ablated groups. This demonstrates persistence of the multidye theranostic nanoparticles within the tumor over 9 days.

Note: Error bars shown represent the standard error of the mean for each measurement.

Abbreviation: MDT-NPs, multidye theranostic nanoparticles.
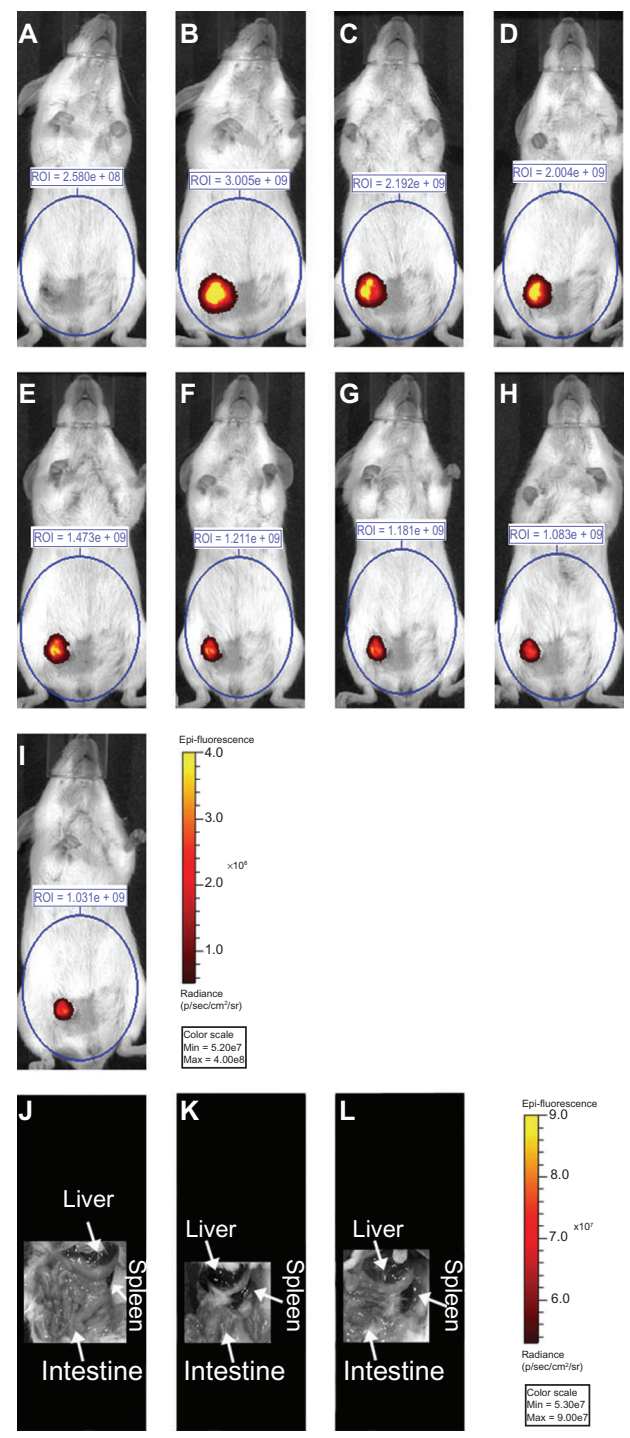

Figure 3 Fluorescence image $(710 \mathrm{~nm}$ excitation/820 nm emission) of a mouse (A) before direct intratumoral multidye theranostic nanoparticle injection, (B) after injection, and $(\mathbf{C})$ after photothermal ablation on day one. This representative mouse underwent daily photothermal ablation (multidye theranostic nanoparticle/ four total ablations) with subsequent fluorescent imaging on (D) day two, (E) day three, and (F) day four. The mouse was further imaged on $(\mathbf{G})$ day five, $(\mathbf{H})$ day seven, and (I) day nine to determine fate of multidye theranostic nanoparticles after photothermal ablation. Region of interest is quantified in photons/second. Image acquisition settings: excitation filter $=710 \mathrm{~nm}$, emission filter $=820 \mathrm{~nm}$, binning = small, field of view $=13.2 \mathrm{~cm}$, f-stop $=2$, exposure $=(\mathbf{A}) \mathrm{I},(\mathbf{B}) 3,(\mathbf{C}) 3$, (D) 3, (E) 3, (F) 4, (G) 5, (H) 6, and (I) 6 seconds. Fluorescent in-situ image (7I0 nm excitation/820 nm emission) of a representative euthanized mouse (J) multidye theranostic nanoparticle/no ablation, (K) multidye theranostic nanoparticle/four ablations, and (L) control/four ablations.

Notes: There is no accumulation of multidye theranostic nanoparticles observed in other organs including the liver and spleen. Image acquisition settings: excitation filter $=710 \mathrm{~nm}$, emission filter $=820 \mathrm{~nm}$, binning $=$ medium, field of view $=13.2 \mathrm{~cm}$, f-stop $=2$, exposure $=\mathrm{I}$ second.

Figure 3 is a fluorescence imaging sequence over a time course of 9 days of a representative mouse from the MDT$\mathrm{NP} /$ four ablations group. After 9 days, MDT-NPs retained high fluorescence imaging capacity and remained within the tumor. In order to determine if MDT-NPs were accumulating 
in reticuloendothelial organs (liver and spleen) following photothermal ablation therapy, whole animal organ in situ fluorescence imaging was performed following primary tumor removal on day nine. No MDT-NPs were detected beyond the intratumoral injection site in these mice (Figure 3).

The persistence of MDT-NPs within the tumor for 9 days following administration suggests the possibility for fractionated image-guided photothermal therapy after a single administration of MDT-NPs to a tumor. Increasing the number of ablation fractions corresponded to a significant $(P=0.026)$ increase in treatment effect as assessed by gross tumor inspection on experiment day nine (Figures 4 and 5). Control groups demonstrated no effect from irradiation, and tumors in the MDT-NP treated/no ablation group were unaltered by the presence of MDT-NPs (Figure 5). Histologically, a congruent pattern was observed: no evidence of thermal injury or other tissue damage was noted in control mice (Figure 6A), whereas mice treated with MDT-NPs and exposed to four fractionated photothermal ablations demonstrated significant thermal injury with coagulative tumor necrosis, hemorrhage, and edema (Figure 6B).

\section{Discussion}

For fractionated photothermal therapy to be possible, photothermal NPs must maintain their intratumoral location following photothermal activation. To the authors' knowledge, the intratumoral fate of photothermal NPs following one or more photothermal treatments has not been previously well characterized. MDT-NPs are a novel tool which has allowed the intratumoral fate of photothermal NPs following photoablation to be studied. Multimodal MDT-NPs can easily be both tracked using fluorescence imaging and repeatedly excited for photoablation with a NIR laser.

In the reported experiments, MDT-NPs were clearly able to be imaged within the tumors following one or more photothermal treatments. The presence of MDT-NPs within the tumor following photothermal treatments (as demonstrated by NIR imaging) and their ability to be repeatedly heated in vitro (Figure 1C) suggests that they may be used for additional photothermal treatments (fractionated photothermal therapy). The ability to image MDT-NPs within the tumor over time can be used to guide fractionated photothermal therapy.

It was observed that MDT-NPs remain in the intratumoral position for days following photothermal treatment in the 4T1 murine mammary carcinoma model. It is possible that the kinetics of nanomaterial extravasation are different for other tumor types with different lymphatic and vascular patterns. This would be a subject worthy of further investigation.

Intratumoral persistence and function of MDT-NPs following photoablation may allow the use of lower laser

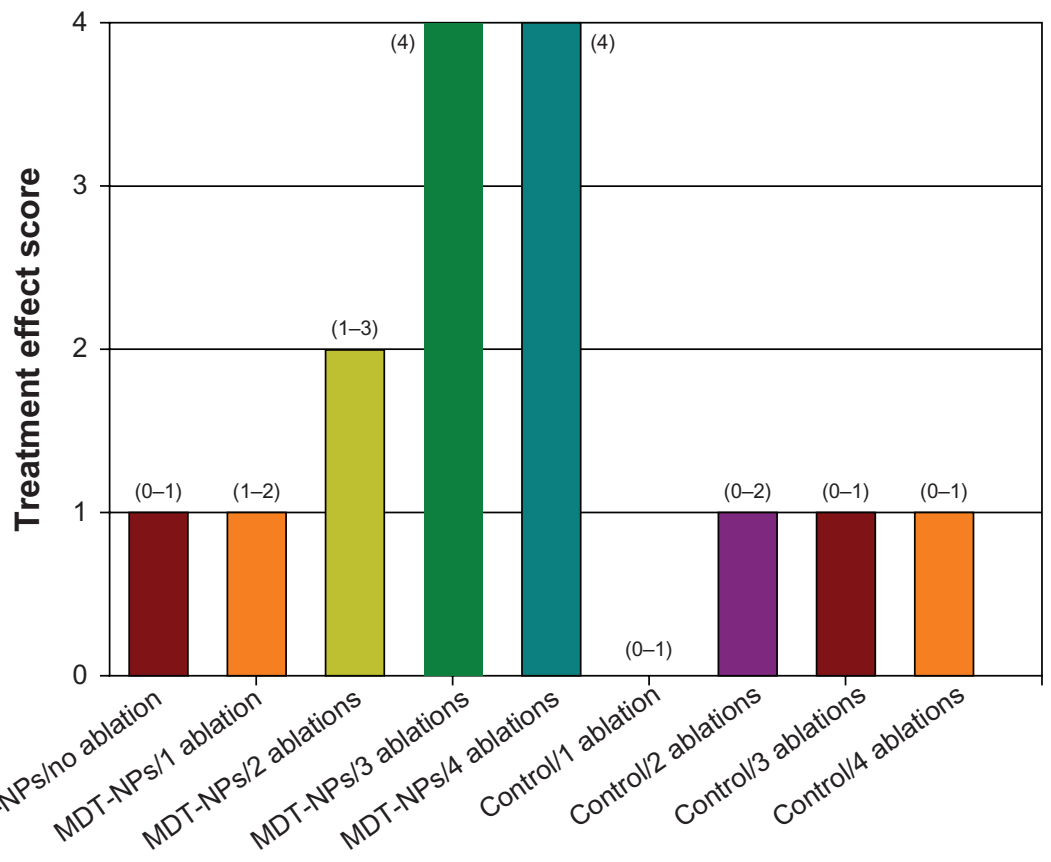

Figure 4 Graph of tumor treatment score in experimental and control mice. Tumor treatment effect was scored in a blinded fashion using a scale of zero (no treatment effect) to four (maximal treatment effect) as determined on experiment day nine.

Notes: Median values are shown with range of values shown in parentheses above the bars. There was a statistical difference in treatment effect score in the treatment groups $(P=0.026)$.

Abbreviation: MDT-NPs, multidye theranostic nanoparticles. 

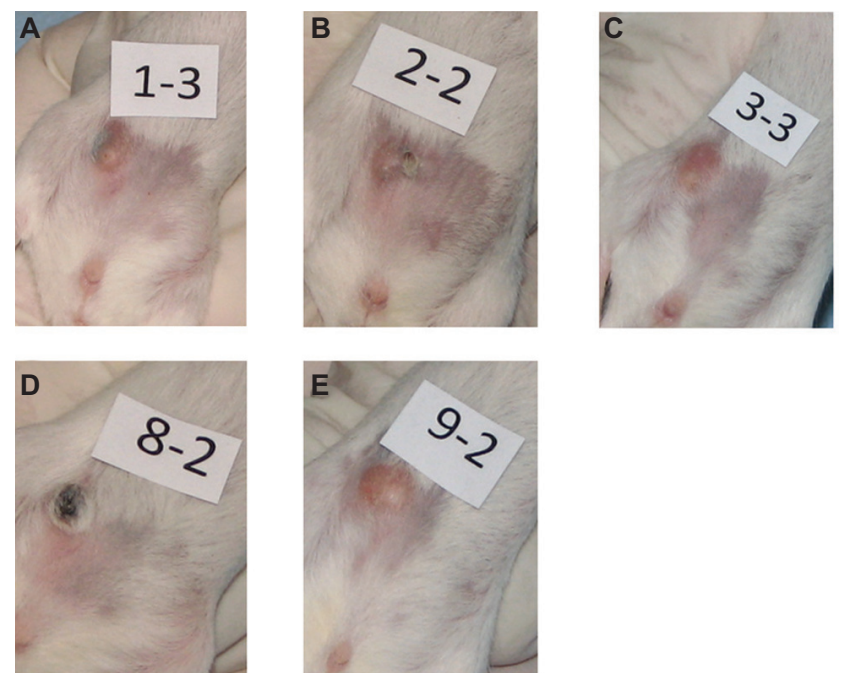

Figure $\mathbf{5}$ Digital photographs of tumors from representative mice from each group taken at 9 days of (A) multidye theranostic nanoparticle/no ablation, (B) multidye theranostic nanoparticle/one ablation, (C) control/one ablation, (D) multidye theranostic nanoparticle/four ablations, and (E) control/four ablations. There is significantly greater tumor destruction seen in the sequentially ablated tumor (D) compared to mice receiving one ablation (B) or control mice (A, C, and $\mathbf{E})$.

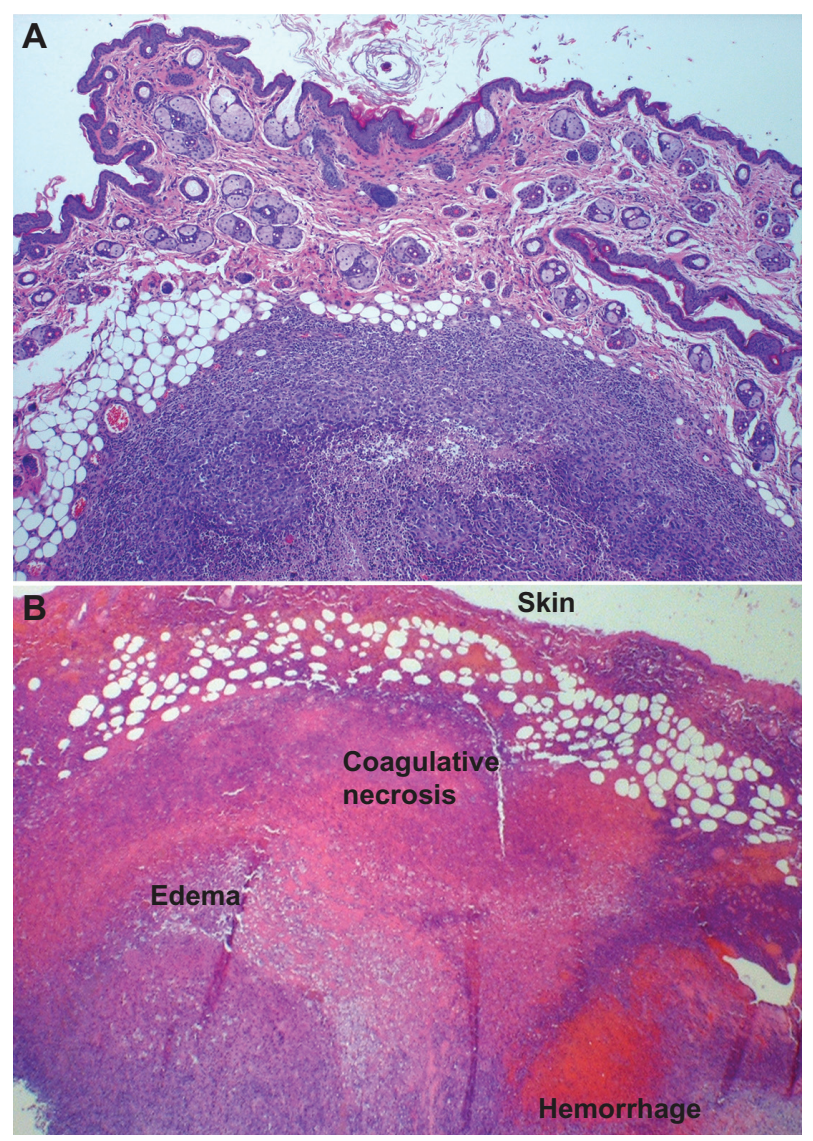

Figure 6 (A) Hematoxylin and eosin stained tumor section of a representative control/four ablations mouse, $4 \times$. There is no evidence of thermal injury or other tissue damage; a viable tumor nodule is clearly demarcated with a healthy skin surface. (B) Hematoxylin and eosin stained tumor section of multidye theranostic nanoparticle/four ablations, $2 \times$. Thermal injury is evident with coagulative tumor necrosis, hemorrhage, and edema in the treated tissues. intensities to treat tumors compared to single ablation strategies. It is noteworthy that in the present study, very low laser intensity $\left(625 \mathrm{~mW} / \mathrm{cm}^{2}\right)$ was required to achieve fractionated photothermal ablation compared to previous reports of photothermal ablation $\left(4 \mathrm{~W} / \mathrm{cm}^{2}\right){ }^{1,10}$ The minimal number of ablations needed to achieve complete tumor destruction is not investigated in the present study and very likely depends on many factors, such as tumor type, tumor size, tumor location, nanoparticle construct (eg, NIR-absorbing component used for ablation), intratumoral distribution of NPs, and concentration of MDT-NPs within tumors. ${ }^{17}$ Such investigations would represent the next step in advancing the concept of fractionated tumor photothermal ablation toward clinical application. The theoretical advantages of fractionated photothermal therapy are evident. Foremost, if complete tumor destruction is not obtained on the initial photothermal treatment, then subsequent successive treatments can be performed using the fractionated approach. Absolute dosing of nanomaterials for photothermal therapy may be minimized with the fractionated treatment paradigm.

\section{Conclusion}

MDT-NPs are unique nanomaterials that maintain their fluorescent and photothermal properties after photoactivation. In the 4T1 murine breast cancer model, MDT-NPs remain within the tumor following photothermal treatments. It has been demonstrated MDT-NPs can therefore be utilized as mediators of fractionated photothermal therapy. Fractionated treatments can be guided by the ability to image MDT-NPs within the tumor using fluorescence imaging techniques.

The concept of image-guided, fractionated photothermal ablation is one which has the potential to expand the efficacy of photothermal therapy for clinical application while minimizing the morbidity of cancer care. This new paradigm should allow photothermal treatments to be delivered and modulated depending on tumor response to achieve the desired clinical effect.

\section{Acknowledgments}

This work was supported by the Florida Department of Health, Bankhead Coley Biomedical Research Program, and United States Department of Defense Breast Cancer Research Program.

\section{Disclosure}

The authors report no conflicts of interest in this work. 


\section{References}

1. Hirsch LR, Stafford RJ, Bankson JA, et al. Nanoshell-mediated near-infrared thermal therapy of tumors under magnetic resonance guidance. Proc Natl Acad Sci U S A. 2003;100(23):13549-13554.

2. Loo C, Lowery A, Halas N, West J, Drezek R. Immunotargeted nanoshells for integrated cancer imaging and therapy. Nano Lett. 2005;5(4):709-711.

3. Huang X, El-Sayed IH, Qian W, El-Sayed MA. Cancer cell imaging and photothermal therapy in the near-infrared region by using gold nanorods. J Am Chem Soc. 2006;128(6):2115-2120.

4. Sharma P, Brown SC, Bengtsson N, et al. Gold-speckled multimodal nanoparticles for noninvasive bioimaging. Chem Mater. 2008;20(19):6087-6094.

5. Krishna V, Singh A, Sharma P, et al. Polyhydroxy fullerenes for noninvasive cancer imaging and therapy. Small. 2010;6(20):2236-2241.

6. Nanospectra Biosciences, Inc Pilot study of aurolase(tm) therapy in refractory and/or recurrent tumors of the head and neck. In: ClinicalTrials.gov [website on the Internet]. Bethseda, MD: US National Library of Medicine; 2009 [updated October 4, 2011]. Available from: http://clinicaltrials.gov/ct2/show/NCT00848042. NLM identifier: NCT00848042. Accessed December 13, 2011.

7. Sharma P, Brown SC, Singh A, et al. Near-infrared absorbing and luminescent gold speckled silica nanoparticles for photothermal therapy. J Mater Chem. 2010;20(25):5182-5185.

8. El-Sayed IH, Huang X, El-Sayed MA. Selective laser photo-thermal therapy of epithelial carcinoma using anti-EGFR antibody conjugated gold nanoparticles. Cancer Lett. 2006;239(1):129-135.

9. Torti SV, Byrne F, Whelan O, et al. Thermal ablation therapeutics based on $\mathrm{CN}(\mathrm{x})$ multi-walled nanotubes. Int J Nanomedicine. 2007;2(4):707-714.
10. Burke A, Ding X, Singh R, et al. Long-term survival following a single treatment of kidney tumors with multiwalled carbon nanotubes and near-infrared radiation. Proc Natl Acad Sci U S A. 2009;106(31):12897-12902.

11. Chen J, Wang D, Xi J, et al. Immuno gold nanocages with tailored optical properties for targeted photothermal destruction of cancer cells. Nano Lett. 2007;7(5):1318-1322.

12. Shashkov EV, Everts M, Galanzha EI, Zharov VP. Quantum dots as multimodal photoacoustic and photothermal contrast agents. Nano Lett. 2008;8(11):3953-3958.

13. Gobin AM, Lee MH, Halas NJ, James WD, Drezek RA, West JL. Near-infrared resonant nanoshells for combined optical imaging and photothermal cancer therapy. Nano Lett. 2007;7(7):1929-1934.

14. Lu W, Melancon MP, Xiong C, et al. Effects of photoacoustic imaging and photothermal ablation therapy mediated by targeted hollow gold nanospheres in an orthotopic mouse xenograft model of glioma. Cancer Res. 2011;71(19):6116-6121.

15. Jin Y, Gao X. Plasmonic fluorescent quantum dots. Nat Nanotechnol. 2009;4(9):571-576.

16. Singh AK, Hahn MA, Gutwein LG, et al. Multi-dye theranostic nanoparticle platform for bioimaging and cancer therapy. Int $J$ Nanomedicine. Forthcoming.

17. Jang B, Kim YS, Choi Y. Effects of gold nanorod concentration on the depth-related temperature increase during hyperthermic ablation. Small. 2011;7(2):265-270
International Journal of Nanomedicine

\section{Publish your work in this journal}

The International Journal of Nanomedicine is an international, peerreviewed journal focusing on the application of nanotechnology in diagnostics, therapeutics, and drug delivery systems throughou the biomedical field. This journal is indexed on PubMed Central, MedLine, CAS, SciSearch ${ }^{\circledR}$, Current Contents ${ }^{\circledR} /$ Clinical Medicine,

\section{Dovepress}

Journal Citation Reports/Science Edition, EMBase, Scopus and the Elsevier Bibliographic databases. The manuscript management system is completely online and includes a very quick and fair peer-review system, which is all easy to use. Visit http://www.dovepress.com/ testimonials.php to read real quotes from published authors. 